\title{
碘化钠-三苯基膦介导的光氧化还原醛亚胺烷基化
}

\author{
郡子宴周庆丽王建成汤苗沈悦海*
}

(昆明理工大学生命科学与技术学院 药学与制药工程中心 昆明 650500)

\begin{abstract}
摘要 电子给受体 $(\mathrm{EDA})$ 络合物参与的光氧化还原反应近十年来备受关注. 新近发现的碘化钠-三苯基膦- $N$-羟基邻苯 二甲酰亚胺㜋酸酯 EDA 络合物已被用于发展多种净氧化还原中性的光反应. 本工作将该 EDA 络合物用于建立净还原 性光反应体系, 发展了无需光敏剂的可见光促进的醛亚胺自由基加成反应. 该反应不仅能以高产率得到仲、叔、 $\alpha$-杂原 子取代烷基自由基加成产物，而且通常反应性不佳的伯烷基自由基以及富电子或仲苄基自由基均能得到较高产率，为 合成非天然氨基酸和胺类化合物提供了高效可靠的新方法.
\end{abstract}

关键词＼cjkstart光氧化还原; 酫亚胺; 电子给受体络合物

\section{Sodium lodide-Triphenylphosphine-Mediated Photoredox Alkylation of Aldimines}

\author{
Shao, Ziyan Zhou, Qingli Wang, Jiancheng Tang, Rui Shen, Yuehai* \\ (Center for Pharmaceutical Sciences and Engineering, Faculty of Life Science and Technology, Kunming University of \\ Science and Technology, Kunming 650500)
}

\begin{abstract}
The photoredox reactions involving electron donor-acceptor (EDA) complexes have attracted significant attentions in the last decade. Very recently, the sodium iodide-triphenylphosphine- $N$-acyloxyphthalimide EDA complex has been discovered and employed in developing several net redox-neutral photoreactions. Herein, the EDA complex has been applied in a net reductive setup for the first time to establish a photoredox alkylation of aldimines without photosensitizer. The reaction affords not only high yields for secondary, tertiary and $\alpha$-heterosubstituted alkyl radicals, but also high yields for primary alkyl radicals, and moderate to high yields for electron-rich or secondary benzylic radicals, two difficult groups of radicals in previous studies. This work provides an efficient and reliable approach for the synthesis of unnatural amino acids and amines. Keywords photoredox; aldimine; electron donor-acceptor complex
\end{abstract}

可见光引发的光氧化还原反应是近年来快速发展 的有力合成手段 ${ }^{[1-3]}$. 有别于能量较高的紫外线可破坏 多种共价键的局限, 可见光较为温和, 能高选择性地产 生自由基 ${ }^{[4]}$, 适用范围更加广泛. 除使用有机光敏剂 ${ }^{[5]}$ 和过渡金属络合物光敏剂 ${ }^{[6]}$ 的较为传统的反应外, 近年 来基于电子给受体(electron donor-acceptor, EDA)络合物 的光氧化还原反应成为研究热点 ${ }^{[7-8]}$. EDA 络合物由富 电子和缺电子反应物组合形成, 各成分本身并不吸收可 见光, 但结合后具备可见光吸收活性, 可在特定波长的 光照射下发生电子转移, 因此无需光敏剂即可启动自由 基反应.
2019 年, 傅尧和尚睿小组 ${ }^{[9]}$ 报道了由 $\mathrm{NaI}$ 、三苯基 膦 (TPP) 和 $N$ - 羟基 邻 苯二甲 酰 亚胺 $(N$-hydroxyphthalimide, NHPI)羧酸酯形成的三组分 EDA 络合物介 导的光氧化还原反应，建立了烯醇硅梄烷基化和对映选 择性 Minisci 反应等净氧化还原中性(net redox-neutral) 的体系(Scheme 1a). 随后, 这一 EDA 络合物光反应体 系受到广泛关注，已被用于 1,6-烯炔的加成环化 ${ }^{[10] 、 2-~}$ 异氧基联苯的自由基环化 ${ }^{[11]}$ 、烯烃烷基化 ${ }^{[12]}$ 和烯酸的脱 羧烷基化 ${ }^{[12-13]}$ 等反应(Scheme 1b). 最近, 类似的碘盐 EDA 络合物也有报道, 李金恒和李杨小组 ${ }^{[14]}$ 发展了 KI三环己基膦-NHPI 酯络合物介导的 1,7-烯炔加成环化反

\footnotetext{
* Corresponding author. E-mail: yuehaishen@kust.edu.cn Received February 22, 2021; revised April 2, 2021; published online April 29, 2021.

Project supported by the National Natural Science Foundation of China (No. 21662022), the Ronald J Quinn AM Academician Workstation and the Program for Innovative Research Team (in Science and Technology) in Universities of Yunnan Province (No. 2019IC003).

国家自然科学基金(No. 21662022)、Ronald J. Quinn AM 院士工作站和云南省教育厅高校创新团队(No. 2019IC003)资助项目.
} 
应, 陈祥雨和汪志祥小组 ${ }^{[15]}$ 报道了 $\mathrm{NaI}$-氮杂环卡宾-吡 啶鎓盐络合物介导的 $\alpha$-碘代反应和 $\mathrm{NaI}-N, N$-二甲基乙 酰胺一吡啶鎓盐络合物介导的 $\alpha$-烯基化反应.

(a) Discovery of Nal-TPP-NHPI ester EDA complex (Fu and Shang, 2019)

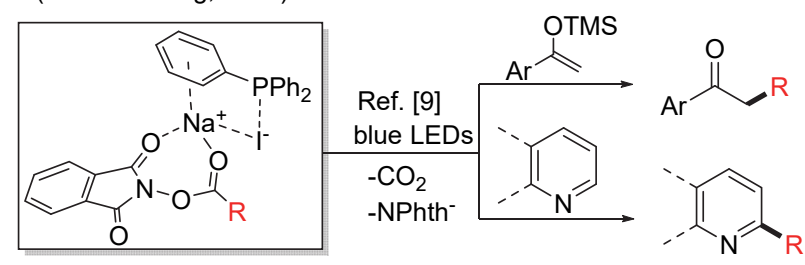

(b) Recent applications (net redox-neutral)
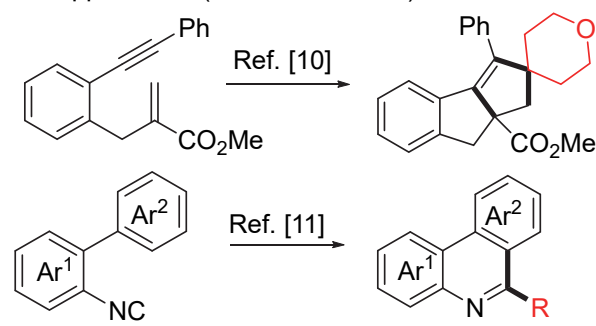

$$
\underset{\mathrm{CO}_{2} \mathrm{H} \stackrel{\text { Ref. [12] }}{\longrightarrow} \text { [12, 13] }}{\longrightarrow}
$$

(c) This work (net reductive)

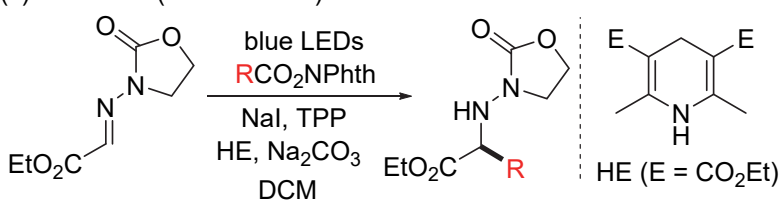

图式 1 NaI-TPP-NHPI 酯的光氧化还原反应

Scheme 1 Photoredox reactions of NaI-TPP-NHPI ester EDA complex

亚胺的还原型自由基反应在胺类化合物的合成方 法中占有独特的地位 ${ }^{[16]}$. 经典方法主要为三烷基嗍、二 烷基锌等引发的自由基加成, 而光氧化还原方法是近年 来比较热门的话题 ${ }^{[17]}$, 主要机理途径可分为从非亚胺 的前体试剂经单电子转移 (single electron transfer, SET) ${ }^{[18]}$ 或氢原子转移(hydrogen atom transfer, HAT) ${ }^{[19]}$ 产 生自由基, 以及从亚胺经质子偶合电子转移 (protoncoupled electron transfer, PCET)产生 $\alpha$-氨基自由基 ${ }^{[20]}$, 但其中鲜有 EDA 络合物反应的报道. 2019年, 俞寿云小 组 ${ }^{[8 \mathrm{e}]}$ 报道了亚胺和 $\alpha$-酮酸的 EDA 络合物, 光照下发生 $\alpha$-氨基自由基和羰基自由基的偶联形成 $\alpha$-氨基酮. 我们 近期开展了醛亚胺净还原性自由基加成合成仲烷基胺 的工作, 分别建立了 $\mathrm{HE}$ 供氢 $\mathrm{Et}_{3} \mathrm{~B}$ 引发的烷基化 ${ }^{[21]}$ 和 $\mathrm{Ru}(\mathrm{bpy}){ }_{3} \mathrm{Cl}_{2}$ 催化可见光介导的脱羧烷基化 ${ }^{[22]}$. 在此基 础上, 通过 NaI-TPP-NHPI 酯 EDA 络合物的光氧化还原 过程引发自由基, 首次建立了该 EDA 络合物的净还原 性光反应体系, 以高产率实现了醛亚胺加成, 并发现可
用于通常反应性较差的伯烷基和茮基自由基的反应 (Scheme 1c).

\section{1 结果与讨论}

基于前期工作 ${ }^{[22]}$ 和相关报道 ${ }^{[9]}$, NaI-TPP-NHPI 酯 EDA 络合物可作为自由基来源，结合适当的还原性试 剂, 发展亚胺加成反应. 原则上, 该 EDA 络合物经可见 光照射发生单电子转移(SET)和 NHPI 酯碎裂, 产生的自 由基可对缺电子亚胺加成生成氮自由基，再经氢原子转 移(HAT)得到预期加成产物(Scheme 2).

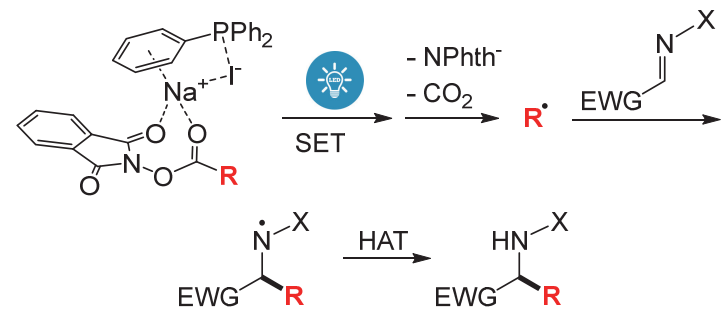

图式 2 设想的反应历程

Scheme 2 Proposed reaction pathway

在条件笁选与优化中, 以乙醛酸乙酯亚胺 $(\mathbf{1 a})$ 和 NHPI 酯 $\mathbf{2 a}$ 的组合作为模板反应, 建立了最优条件 (Entry 1, 表 1), 以 Hantzsch 酯(HE)作为氢供体试剂、二 氯甲烷(DCM)作为溶剂、 $\mathrm{Na}_{2} \mathrm{CO}_{3}$ 作为碱时, 室温下光照 $10 \mathrm{~h}$ 得到接近定量的 $96 \%$ 产率. $\mathrm{NaI}$ 和 TPP 的用量分别 确定为 1.5 equiv. 和 0.3 equiv. (Entries 1 5). HE 的用量 以 1.0 equiv. 为佳(Entries 1, 6 7), 与需要 1.5 equiv. 的 $\mathrm{Ru}(\mathrm{bpy}){ }_{3} \mathrm{Cl}_{2}$ 催化体系 ${ }^{[22]}$ 不同. 反应在乙腈、丙酮、 $\mathrm{THF}$ 和 1,2-二氯乙烷中产率均明显下降(Entries 8 11). 无机 碱 $\mathrm{Na}_{2} \mathrm{CO}_{3}$ 具有较为明显的促进作用, 无 $\mathrm{Na}_{2} \mathrm{CO}_{3}$ 时亚胺 $1 \mathbf{a}$ 无法转化完全(Entries 1, 12), 如将 $\mathrm{Na}_{2} \mathrm{CO}_{3}$ 改为活化 分子篮和无水 $\mathrm{Na}_{2} \mathrm{SO}_{4}$ 则产率下降(Entries 13,14). 然而, 无机碱中的碱金属离子也会影响反应，将 $\mathrm{Na}_{2} \mathrm{CO}_{3}$ 换成 同族的 $\mathrm{K}_{2} \mathrm{CO}_{3}$ 和 $\mathrm{Cs}_{2} \mathrm{CO}_{3}$ 显著抑制反应(Entries 15, 16). 类似的是, $\mathrm{NaI}$ 如改为 $\mathrm{KI}$ 也造成产率下降(Entry 17). 上 述金属离子效应与傅尧和尚睿小组的结果 ${ }^{[9]}$ 相同. 此外, 体系中加入少量碘单质 ${ }^{[9]}$ 也可明显抑制反应(Entry 18).

同时进行了多个对照实验(Entries 19～24). 无 NaI、 无 TPP 和无 NaI-TPP 的对照反应的结果类似, 均得到 20\% 30\%产率(Entries 19 21), 应来源于 HE 吸光引起 的背景反应 ${ }^{[18 \mathrm{~d}, 22-23]}$ ，表明 NaI-TPP-NHPI 酯 EDA 络合物 应为体系中的主要光敏物质. 无 HE (Entry 22)或避光 (Entry 23)条件下反应无法进行, 而且加入 TEMPO 能完 全抑制反应，表明此体系确为光催化引发自由基的反 应, $\mathrm{HE}$ 在其中为供氢试剂. 这些结果证实了我们的机理 设想. 
表 1 条件优化和对照试验

Table 1 Optimization of reaction conditions and control experiments

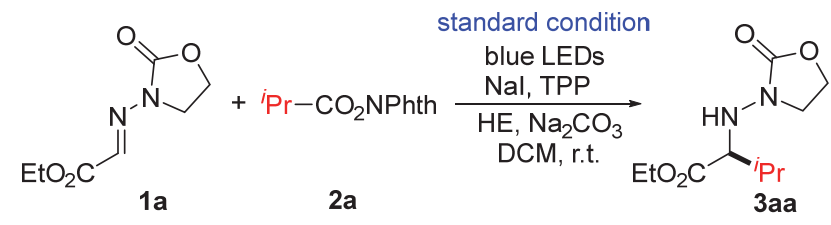

\begin{tabular}{|c|c|c|}
\hline Entry & Variations from the "standard condition" $a$ & Yield $/ \%$ \\
\hline 1 & None & 96 \\
\hline 2 & $\mathrm{NaI}$ ( 0.5 equiv. $)$ & 80 \\
\hline 3 & $\mathrm{NaI}$ (1.0 equiv.) & 84 \\
\hline 4 & ТPP ( 0.2 equiv. $)$ & 86 \\
\hline 5 & ТPP ( 0.5 equiv. $)$ & 95 \\
\hline 6 & HE ( 0.5 equiv.) & 80 \\
\hline 7 & HE (1.5 equiv.) & 96 \\
\hline 8 & In acetonitrile & 23 \\
\hline 9 & In acetone & 35 \\
\hline 10 & In THF & 65 \\
\hline 11 & In 1,2-dichloroethane & 10 \\
\hline 12 & $\mathrm{No} \mathrm{Na}_{2} \mathrm{CO}_{3}$ & 85 \\
\hline 13 & $4 \AA \mathrm{MS}$ instead of $\mathrm{Na}_{2} \mathrm{CO}_{3}$ & 71 \\
\hline 14 & $\mathrm{Na}_{2} \mathrm{SO}_{4}$ instead of $\mathrm{Na}_{2} \mathrm{CO}_{3}$ & 88 \\
\hline 15 & $\mathrm{~K}_{2} \mathrm{CO}_{3}$ instead of $\mathrm{Na}_{2} \mathrm{CO}_{3}$ & 54 \\
\hline 16 & $\mathrm{Cs}_{2} \mathrm{CO}_{3}$ instead of $\mathrm{Na}_{2} \mathrm{CO}_{3}$ & 40 \\
\hline 17 & $\mathrm{KI}$ instead of $\mathrm{NaI}$ & 73 \\
\hline 18 & Add $\mathrm{I}_{2}(0.05$ equiv. $)$ & $<10$ \\
\hline 19 & No TPP & 27 \\
\hline 20 & No NaI & 20 \\
\hline 21 & No TPP and NaI & 25 \\
\hline 22 & No HE & n.r..$^{c}$ \\
\hline 23 & No light & n.r..$^{c}$ \\
\hline 24 & Add TEMPO (5.0 equiv.) & n.r. ${ }^{c}$ \\
\hline
\end{tabular}

${ }^{a}$ The "standard condition": 1a $(0.16 \mathrm{mmol}), \mathbf{2 a}(1.5$ equiv.), HE (1.0 equiv.), $\mathrm{NaI}$ (1.5 equiv.), TPP (0.3 equiv.), $\mathrm{Na}_{2} \mathrm{CO}_{3}$ (2.0 equiv.) in DCM $(0.5 \mathrm{~mL})$, irradiation by blue LEDs $(36 \mathrm{~W}$ ) at room temperature for $10 \mathrm{~h}$ under an argon atmosphere. ${ }^{b}$ Isolated yields. ${ }^{c}$ n.r.: no reaction.

上述最优条件随后用于各类 NHPI 酯, 考查各种类 型自由基的反应性(Scheme 3). 大多数简单羧酸的 NHPI 酯都能得到较好的结果. 对于反应性较好的仲碳自由基 (3aa $\sim 3 a c)$ 和叔碳自由基(3ad 3af)，分离产率在 88\% 以上，吉非罗齐 NHPI 酯的产率为 93\% (3af). $\alpha$-杂原子 取代羧酸 NHPI 酯也可得到较好结果. $\alpha$-氨基取代自由 基普遍反应性优异(3ag 3ak), 产率一般高于 90\%, 仅 $3 \mathrm{ag}$ 稍低 (75\%). 其中, 3ak 的产率 (93\%) 明显高于 $\mathrm{Ru}(\mathrm{bpy})_{3} \mathrm{Cl}_{2}$ 催化的 $67 \%$ 产率 ${ }^{[22]}$. $\alpha$-氧取代自由基的产率 稍低(3al, 78\%). 上述结果与 $\mathrm{Ru}(\mathrm{bpy})_{3} \mathrm{Cl}_{2}$ 催化体系 ${ }^{[22]}$ 基 本一致.

意外的是, 稳定性较弱的伯碳自由基的表现并不明 显逊色(3am 3ar). 在 1.5 equiv. NHPI 酯用量下, 直链
烷基自由基均能获得 80\%以上的产率(3ag 3ap)，而且 末端双键或溴取代影响不大. $\alpha$-碳位阻较大的新戊基自 由基的产率较低(3aq, 56\%)，但甲基自由基能得到 74\% 的较高产率(3ar).

值得指出的是，反应性较弱的苄基类自由基在 $\mathrm{Ru}(\mathrm{bpy})_{3} \mathrm{Cl}_{2}$ 体系中产率很低, 但在本体系中, 除苄基自 由基产率仅 27\% (3as)外, 富电子或仲苠基自由基可得 到较好的结果(3at 3ay). 其中，1-苯基乙基自由基的反 应产率为 $81 \%$, 远高于 $\mathrm{Ru}(\mathrm{bpy})_{3} \mathrm{Cl}_{2}$ 催化得到的 $22 \%$. 不 同于其它羧酸 NHPI 酯的反应，芐基羧酸 NHPI 酯的反 应较难转化完全. 其中, 非甾体抗炎药吲哚美辛 NHPI 酯可实现完全转化，产率较高(3ay，91\%), 而酮洛芬和 荎普生 NHPI 酯的产率较低(3aw, 58\%; 3ax, 52\%).

这一体系也适用于其它类型的亚胺底物，例如 4-三 氟甲基苯甲醛亚胺 $1 \mathrm{~b}$ 和 $O$-苄基肜醚 $1 \mathrm{c}$ 与 $\mathbf{2 a}$ 反应均可 得到 60\% 70\%的较好产率，但 $O$-硅基肟醚 1d 产率较 低(Scheme 4).

此方法可用于天然产物的结构修饰, 将羒基替换为 氨基酸片段 (Scheme 5a). 我去氧胆酸 NHPI 酯与过量亚 胺 $1 \mathrm{a}$ 的反应可得到 $84 \%$ 的高产率. 齐墩果酸和山楂酸 NHPI 酯的反应产物为单一立体构型 ${ }^{[22]}$, 但产率较低. 反应的量级也可进一步放大, 但需要视情况适当延长反 应时间 (Scheme 5b). 在光照强度不变(蓝光 LED, 18 $\mathrm{W} \times 2$ )的克级反应中, 分子量较小的异丁酸 NHPI 酯反 应 $10 \mathrm{~h}$ 即以 $91 \%$ 的高产率得到产物 3aa. 对于自由基片 段分子量较大的产物 3af 和 3ay，反应时间各延长至 39 和 $48 \mathrm{~h}$ 能得到较好的产率.

与 $\mathrm{Ru}(\mathrm{bpy}){ }_{3} \mathrm{Cl}_{2}$ 催化体系 ${ }^{[22]}$ 比较, 本方法同样需要 无机碱促进, 表明 HE 的供氢过程相似; 但由于 NHPI 酯适用范围有显著差异，提示在自由基的产生和加成等 方面有独特之处. 在此或涉及 EDA 络合物与醛亚胺之 间的相互作用，相关细节有待进一步探讨.

\section{2 结论}

将 NaI-TPP-NHP 酯 EDA 络合物用于净还原性光反 应，建立了无需光敏剂的光氧化还原醛亚胺自由基加成 反应. 该反应体系温和、高效，不仅仲、叔、 $\alpha$-杂原子 取代烷基自由基能以高产率得到加成产物，而且通常反 应性不佳的伯烷基自由基以及富电子和仲芐基自由基 也能得到较高产率，为非天然氨基酸和胺类化合物的合 成提供了新的方法.

\section{3 实验部分}

\section{1 仪器与试剂}

${ }^{1} \mathrm{H}$ NMR 和 ${ }^{13} \mathrm{C}$ NMR 谱图通过 Bruker Avance III 


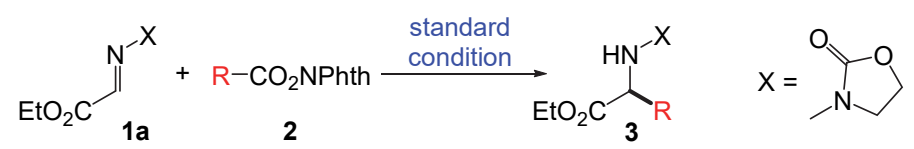


(a) Reaction of natural product NHPI esters
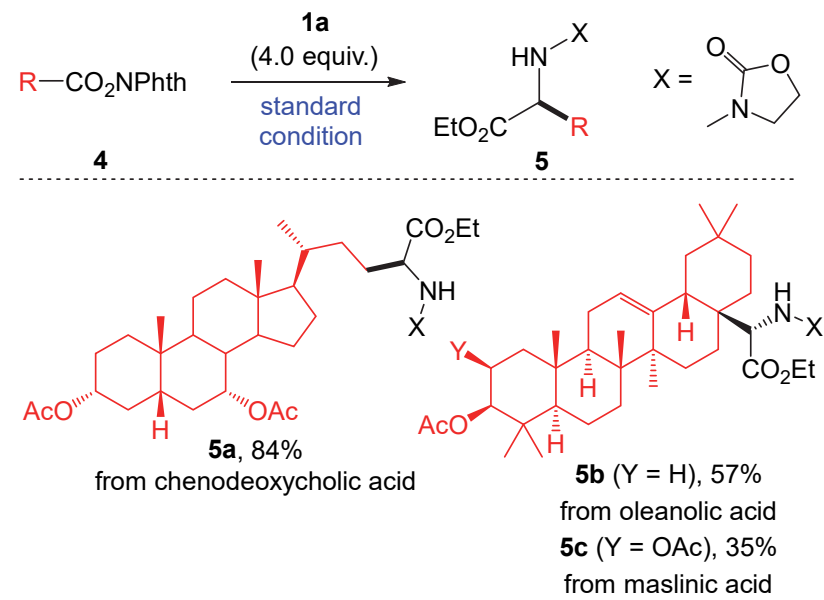

(b) Gram-scale reaction

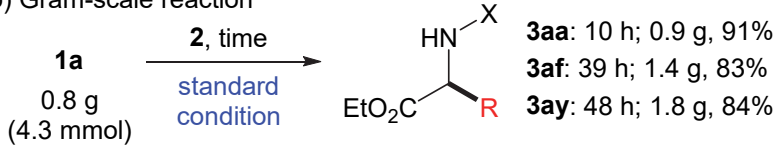

图式 5 天然产物修饰和克级反应

Scheme 5 Natural product modification and gram-scale reaction

下室温摚拌 16 h, TLC 跟踪反应. 反应完成后减压浓缩, 硅胶柱层析(PE/EA)分离, 得到相应的加成产物.

通法 B(化合物 3ba 3da)：反应使用醛亚胺 1 (50 $\mathrm{mg}) 、 \mathrm{NHPI}$ 酯 2a (1.5 equiv.)、NaI (1.5 equiv.)、TPP ( 0.3 equiv.)、 $\mathrm{HE}$ (1.0 equiv.)、 $\mathrm{Na}_{2} \mathrm{CO}_{3}$ (2.0 equiv.) 和无水 $\mathrm{DCM}$ $(0.5 \sim 1.0 \mathrm{~mL})$. 实验操作和后处理、分离纯化与步骤 $\mathrm{A}$ 相同.

通法 C(化合物 5)：反应使用天然产物 NHPI 酯 4 (50 $\mathrm{mg}) 、$ 醛亚胺 1a (4.0 equiv.)、NaI (1.5 equiv.)、TPP (0.3 equiv.)、 $\mathrm{HE}$ (1.0 equiv.)、 $\mathrm{Na}_{2} \mathrm{CO}_{3}$ (2.0 equiv.) 和无水 $\mathrm{DCM}$ $(5 \mathrm{~mL} / \mathrm{mmol} \mathrm{1a})$. 实验操作和后处理、分离纯化与步骤 $\mathrm{A}$ 相同.

6-(2,5-二甲基苯氧基)-3,3-二甲基-2-((2-氧代噁唑 啉-3-基)氨基)己酸乙酯(3af): 采用通法 $\mathrm{A}$, 柱层析洗脱 剂 PE/EA $(V: V=3: 1)$; 无色油状物 $59 \mathrm{mg}$, 产率 93\%. ${ }^{1} \mathrm{H}$ NMR $\left(600 \mathrm{MHz}, \mathrm{CDCl}_{3}\right) \delta: 6.93(\mathrm{~d}, J=7.0 \mathrm{~Hz}, 1 \mathrm{H})$, $6.58(\mathrm{~d}, J=7.0 \mathrm{~Hz}, 1 \mathrm{H}), 6.55(\mathrm{~s}, 1 \mathrm{H}), 4.20 \sim 4.12(\mathrm{~m}, 4 \mathrm{H})$, $3.86(\mathrm{t}, J=6.0 \mathrm{~Hz}, 2 \mathrm{H}), 3.52(\mathrm{t}, J=8.0 \mathrm{~Hz}, 2 \mathrm{H}), 3.31(\mathrm{~s}$, $1 \mathrm{H}), 2.23(\mathrm{~s}, 3 \mathrm{H}), 2.10(\mathrm{~s}, 3 \mathrm{H}), 1.80 \sim 1.72(\mathrm{~m}, 2 \mathrm{H}), 1.56 \sim$ $1.49(\mathrm{~m}, 1 \mathrm{H}), 1.47 \sim 1.39(\mathrm{~m}, 1 \mathrm{H}), 1.23(\mathrm{t}, J=7.0 \mathrm{~Hz}, 3 \mathrm{H})$, 0.97 (s, 3H), 0.93 (s, 3H); $\left.{ }^{13} \mathrm{C} \mathrm{NMR} \mathrm{(150} \mathrm{MHz,} \mathrm{CDCl}_{3}\right) \delta$ : $171.9,157.6,155.9,135.5,129.2,122.4,119.6,110.9$, $69.5,67.1,60.4,59.9,46.2,35.2,35.0,22.9,22.8,22.6$, 20.4, 14.8, 13.2; HRMS (ESI) calcd for $\mathrm{C}_{21} \mathrm{H}_{32} \mathrm{~N}_{2} \mathrm{NaO}_{5}$ $[\mathrm{M}+\mathrm{Na}]^{+}$415.2203, found 415.2201.

2-((2-氧代噁唑啉-3-基)氨基)-5-苯基戊酸乙酯(3ao):
采用通法 A, 柱层析洗脱剂 PE/EA $(V: V=5: 2)$; 无色 油状物 $46 \mathrm{mg}$, 产率 94\%. ${ }^{1} \mathrm{H}$ NMR (600 MHz, $\left.\mathrm{CDCl}_{3}\right) \delta$ : $7.23 \sim 7.18(\mathrm{~m}, 2 \mathrm{H}), 7.13 \sim 7.08(\mathrm{~m}, 3 \mathrm{H}), 4.20 \sim 4.08(\mathrm{~m}$, $4 \mathrm{H}), 3.70$ (t, $J=6.0 \mathrm{~Hz}, 1 \mathrm{H}), 3.61(\mathrm{dd}, J=15.0,7.5 \mathrm{~Hz}$, $1 \mathrm{H}), 3.45(\mathrm{dd}, J=16.0,7.5 \mathrm{~Hz}, 1 \mathrm{H}), 2.58(\mathrm{t}, J=7.0 \mathrm{~Hz}$, $2 \mathrm{H}), 1.75 \sim 1.59(\mathrm{~m}, 4 \mathrm{H}), 1.21(\mathrm{t}, J=7.0 \mathrm{~Hz}, 3 \mathrm{H}) ;{ }^{13} \mathrm{C}$ NMR $\left(150 \mathrm{MHz}, \mathrm{CDCl}_{3}\right) \delta: 171.8,157.9,140.6,127.5$, 127.3, 124.9, 61.2, 60.6, 60.1, 46.8, 34.4, 29.1, 26.0, 13.2; HRMS (ESI) calcd for $\mathrm{C}_{16} \mathrm{H}_{22} \mathrm{~N}_{2} \mathrm{NaO}_{4}[\mathrm{M}+\mathrm{Na}]$ 329.1472 , found 329.1473 .

2-((2-氧代噁唑啉-3-基)氨基)十九酸乙酯(3ap): 采 用通法 $\mathrm{A}$, 柱层析洗脱剂 PE/EA $(V: V=5: 1)$; 白色固 体 $67 \mathrm{mg}$, 产率 97\%. m.p. 63 64 ${ }^{\circ} \mathrm{C} ;{ }^{1} \mathrm{H}$ NMR $(600$ $\left.\mathrm{MHz}, \mathrm{CDCl}_{3}\right) \delta: 4.26 \sim 4.09(\mathrm{~m}, 4 \mathrm{H}), 3.70 \sim 3.65(\mathrm{~m}, 2 \mathrm{H})$, $3.57(\mathrm{dd}, J=16.0,8.0 \mathrm{~Hz}, 1 \mathrm{H}), 1.68 \sim 1.55(\mathrm{~m}, 2 \mathrm{H})$, $1.39 \sim 1.26(\mathrm{~m}, 2 \mathrm{H}), 1.26 \sim 1.14(\mathrm{~m}, 28 \mathrm{H}), 1.23(\mathrm{t}, J=7.0$ $\mathrm{Hz}, 3 \mathrm{H}), 0.81(\mathrm{t}, J=7.0 \mathrm{~Hz}, 3 \mathrm{H}) ;{ }^{13} \mathrm{C}$ NMR $(150 \mathrm{MHz}$, $\left.\mathrm{CDCl}_{3}\right) \delta: 172.0,157.9,61.6,60.6,60.0,46.8,30.9,29.9$, $28.7,28.6,28.6,28.5,28.4,28.4,28.3,24.4,21.7,13.2$, 13.1; HRMS (ESI) calcd for $\mathrm{C}_{24} \mathrm{H}_{46} \mathrm{~N}_{2} \mathrm{NaO}_{4}[\mathrm{M}+\mathrm{Na}]^{+}$ 449.3350, found 449.3350.

(2-氧代噁唑啉-3-基)苯丙氨酸乙酯(3as): 采用通法 $\mathrm{A}$, 柱层析洗脱剂 $\mathrm{PE} / \mathrm{EA}(V: V=3: 1)$; 白色固体 12 $\mathrm{mg}$, 产率 27\%. m.p. $71 \sim 72{ }^{\circ} \mathrm{C}$; ${ }^{1} \mathrm{H}$ NMR $(600 \mathrm{MHz}$, $\left.\mathrm{CDCl}_{3}\right) \delta: 7.27 \sim 7.14(\mathrm{~m}, 5 \mathrm{H}), 4.40$ (bs, $\left.1 \mathrm{H}\right), 4.16 \sim 4.02$ $(\mathrm{m}, 5 \mathrm{H}), 3.64 \sim 3.55(\mathrm{~m}, 1 \mathrm{H}), 3.39(\mathrm{q}, J=8.5 \mathrm{~Hz}, 1 \mathrm{H})$, 3.05 (dd, $J=14.5,6.0 \mathrm{~Hz}, 1 \mathrm{H}), 2.85(\mathrm{dd}, J=14.0,9.0 \mathrm{~Hz}$, $1 \mathrm{H}), 1.14(\mathrm{t}, \quad J=7.0 \mathrm{~Hz}, 3 \mathrm{H}) ;{ }^{13} \mathrm{C}$ NMR $(150 \mathrm{MHz}$, $\left.\mathrm{CDCl}_{3}\right) \delta: 171.2,157.8,135.2,128.1,127.6,126.0,62.2$, 60.6, 60.2, 46.7, 36.5, 13.0; HRMS (ESI) calcd for $\mathrm{C}_{14} \mathrm{H}_{18} \mathrm{~N}_{2} \mathrm{NaO}_{4}[\mathrm{M}+\mathrm{Na}]^{+}$301.1159, found 301.1157.

3-(3,4-二甲氧基苯基)-2-((2-氧代噁唑啉-3-基)氨基) 丙酸乙酯(3at): 采用通法 $\mathrm{A}$, 柱层析洗脱剂 PE/EA $(V$ : $V=2: 1)$; 无色油状物 $42 \mathrm{mg}$, 产率 76\%. ${ }^{1} \mathrm{H}$ NMR $(600$ $\left.\mathrm{MHz}, \mathrm{CDCl}_{3}\right) \delta: 6.87 \sim 6.77(\mathrm{~m}, 3 \mathrm{H}), 4.28 \sim 4.13(\mathrm{~m}, 5 \mathrm{H})$, $3.89(\mathrm{~s}, 3 \mathrm{H}), 3.86(\mathrm{~s}, 3 \mathrm{H}), 3.76 \sim 3.70(\mathrm{~m}, 1 \mathrm{H}), 3.52(\mathrm{q}, J=$ $8.5 \mathrm{~Hz}, 1 \mathrm{H}), 3.10$ (dd, $J=14.0,6 \mathrm{~Hz}, 1 \mathrm{H}), 2.85$ (dd, $J=$ $14.0,9.0 \mathrm{~Hz}, 1 \mathrm{H}), 1.25(\mathrm{t}, J=7.0 \mathrm{~Hz}, 3 \mathrm{H}) ;{ }^{13} \mathrm{C}$ NMR $(150$ $\left.\mathrm{MHz}, \mathrm{CDCl}_{3}\right) \delta: 172.4,158.8,149.0,148.0,128.3,121.2$, $111.9,111.1,62.9,61.7,61.2,55.9,55.8,47.7,36.9,14.1$; HRMS (ESI) calcd for $\mathrm{C}_{16} \mathrm{H}_{22} \mathrm{~N}_{2} \mathrm{NaO}_{6}[\mathrm{M}+\mathrm{Na}]^{+}$ 361.1370 , found 361.1372 .

2-((2-氧代噁唑啉-3-基)氨基)-3-苯基丁酸乙酯 (3au): 采用通法 $\mathrm{A}$, 柱层析洗脱剂 $\mathrm{PE} / \mathrm{EA}(V: V=3$ : 1); 白色固体 $38 \mathrm{mg}, d r 1.7: 1$, 产率 81\%. m.p. 90 
$92{ }^{\circ} \mathrm{C} ;{ }^{1} \mathrm{H}$ NMR $\left(600 \mathrm{MHz}, \mathrm{CDCl}_{3}\right) \delta: 7.31 \sim 7.13(\mathrm{~m}$, $5 \mathrm{H}),(4.22 \sim 4.07)(\mathrm{m}, 3 \mathrm{H}),(4.06 \sim 4.01,3.99 \sim 3.94)(\mathrm{m}$, $1 \mathrm{H}),(3.93,3.78)(\mathrm{d}, J=8.5 \mathrm{~Hz}, 1 \mathrm{H}), 3.49 \sim 3.42(\mathrm{~m}, 1 \mathrm{H})$, $(3.35,3.20)(\mathrm{q}, J=8.5 \mathrm{~Hz}, 1 \mathrm{H}),(3.13 \sim 3.07,3.01 \sim 2.94)$ $(\mathrm{m}, 1 \mathrm{H}),(1.33,1.25)(\mathrm{d}, J=7.0 \mathrm{~Hz}, 3 \mathrm{H}),(1.23,1.02)(\mathrm{t}$, $J=7.0 \mathrm{~Hz}, 3 \mathrm{H}) ;{ }^{13} \mathrm{C} \mathrm{NMR}\left(150 \mathrm{MHz}, \mathrm{CDCl}_{3}\right) \delta:(171.5$, 171.2), 157.5, (140.9, 140.8), (127.8, 127.4), (126.8, 125.9), (126.6, 126.3), (67.6 67.0), (60.6, 60.5), (60.2, 60.0), (46.4, 46.3), (40.7, 40.1), (18.2, 15.2), (13.2, 12.9); HRMS (ESI) calcd for $\mathrm{C}_{15} \mathrm{H}_{20} \mathrm{~N}_{2} \mathrm{NaO}_{4} \quad\left[\mathrm{M}+\mathrm{Na}{ }^{+}\right.$ 315.1315 , found 315.1316 .

3-(4-异丁基苯基)-2-((2-氧代啞唑啉-3-基)氨基)丁 酸乙酯(3av): 采用通法 $\mathrm{A}$, 柱层析洗脱剂 PE/EA $(V$ : $V=3: 1$ ); 无色油状物 $45 \mathrm{mg}, d r 1: 1$, 产率 $79 \%$. ${ }^{1} \mathrm{H}$ NMR $\left(600 \mathrm{MHz}, \mathrm{CDCl}_{3}\right) \delta: 7.14 \sim 7.07(\mathrm{~m}, 2 \mathrm{H}), 7.06 \sim$ $6.98(\mathrm{~m}, 2 \mathrm{H}), 4.57(\mathrm{bs}, 1 \mathrm{H}), 4.21 \sim 4.15(\mathrm{~m}, 1 \mathrm{H}), 4.14 \sim$ $4.00(\mathrm{~m}, 2 \mathrm{H}), 3.99 \sim 3.92(\mathrm{~m}, 1 \mathrm{H}),(3.90,3.76)(\mathrm{d}, J=8.5$ $\mathrm{Hz}, 1 \mathrm{H}), 3.49 \sim 3.41(\mathrm{~m}, 1 \mathrm{H}),(3.36,3.19)(\mathrm{q}, J=9.5 \mathrm{~Hz}$, $1 \mathrm{H}),(3.10 \sim 3.04,2.98 \sim 2.91)(\mathrm{m}, 1 \mathrm{H}), 2.36(\mathrm{~d}, J=7.0$ $\mathrm{Hz}, 2 \mathrm{H}), 1.81 \sim 1.71(\mathrm{~m}, 1 \mathrm{H}),(1.31,1.21)(\mathrm{d}, J=7.0 \mathrm{~Hz}$, $3 \mathrm{H}),(1.23,1.02)(\mathrm{td}, J=7.0,0.5 \mathrm{~Hz}, 3 \mathrm{H}), 0.81$ (t, $J=7.0$ $\mathrm{Hz}, 6 \mathrm{H}) ;{ }^{13} \mathrm{C} \mathrm{NMR}\left(150 \mathrm{MHz}, \mathrm{CDCl}_{3}\right) \delta:(171.6,171.4)$, (157.6, 157.5), (139.6, 139.3), (138.1, 137.9), (128.5, 128.0), (126.5, 126.3), (67.7, 67.1), (60.6, 60.5), (60.1, 59.9), (46.3, 46.2), (44.0, 43.9), (40.3, 39.8), (29.2, 29.1), (21.4, 21.3), (18.2, 15.2), (13.2, 12.9); HRMS (ESI) calcd for $\mathrm{C}_{19} \mathrm{H}_{28} \mathrm{~N}_{2} \mathrm{NaO}_{4}[\mathrm{M}+\mathrm{Na}]^{+}$371.1941, found 371.1941.

3-(3-苯甲酰基苯基)-2-((2-氧代噁唑啉-3-基)氨基) 丁酸乙酯(3aw): 采用通法 $\mathrm{A}$, 柱层析洗脱剂 $\mathrm{PE} / \mathrm{EA}(V$ : $V=3: 1)$; 黄色油状物 $37 \mathrm{mg}, d r 1: 1$, 产率 $58 \%$. ${ }^{1} \mathrm{H}$ NMR (600 MHz, $\left.\mathrm{CDCl}_{3}\right) \delta: 7.77 \sim 7.69(\mathrm{~m}, 2 \mathrm{H}), 7.64(\mathrm{~d}$, $J=11.0 \mathrm{~Hz}, 1 \mathrm{H}),(7.61,7.58)(\mathrm{d}, J=8.0 \mathrm{~Hz}, 1 \mathrm{H}), 7.55 \sim$ $7.50(\mathrm{~m}, 1 \mathrm{H}), 7.49 \sim 7.33(\mathrm{~m}, 4 \mathrm{H}), 4.20 \sim 4.04(\mathrm{~m}, 3 \mathrm{H})$, 3.98 (q, $J=7.0 \mathrm{~Hz}, 1 \mathrm{H}),(3.88,3.77)(\mathrm{d}, J=7.5 \mathrm{~Hz}, 1 \mathrm{H})$, $3.53 \sim 3.43(\mathrm{~m}, 1 \mathrm{H}),(3.38,3.29)(\mathrm{q}, J=8.0 \mathrm{~Hz}, 1 \mathrm{H}),(3.17$, 3.09 ) (q, $J=7.0 \mathrm{~Hz}, 1 \mathrm{H}),(1.35,1.26)(\mathrm{d}, J=7.0 \mathrm{~Hz}, 3 \mathrm{H})$, $(1.21,1.04)(\mathrm{td}, J=7.0,1.0 \mathrm{~Hz}, 3 \mathrm{H}) ;{ }^{13} \mathrm{C} \mathrm{NMR}(150 \mathrm{MHz}$, $\left.\mathrm{CDCl}_{3}\right) \delta$ : (195.59, 195.57), (171.22, 170.97), (157.65, 157.64), (141.41, 141.22), (136.92, 136.65), (136.39, 136.34), (131.55, 131.54), (131.01, 130.74), (129.10, 129.05), (128.33, 128.24), (128.04, 127.82), (127.70, 127.32), (127.35, 127.33), (67.39, 67.01), (60.55, 60.52), (60.31, 60.14), 46.37, (40.55, 40.14), (17.85, 15.41), (13.18, 12.95); HRMS (ESI) calcd for $\mathrm{C}_{22} \mathrm{H}_{24} \mathrm{~N}_{2} \mathrm{NaO}_{5}$ $[\mathrm{M}+\mathrm{Na}]^{+}$419.1577, found 419.1579.
3-(6-甲氧基萗-2-基)-2-((2-氧代噁唑啉-3-基)氨基) 丁酸乙酯 $(\mathbf{3 a x})$ : 采用通法 $\mathrm{A}$, 柱层析洗脱剂 $\mathrm{PE} / \mathrm{EA}(V$ : $V=3: 1)$; 黄色油状物 $31 \mathrm{mg}, d r 1: 1$, 产率 $52 \% .{ }^{1} \mathrm{H}$ NMR $\left(600 \mathrm{MHz}, \mathrm{CDCl}_{3}\right) \delta: 7.68 \sim 7.60(\mathrm{~m}, 2 \mathrm{H}),(7.59$, $7.54)(\mathrm{s}, 1 \mathrm{H}), 7.32$ (ddd, $J=8.5,7.0,2.0 \mathrm{~Hz}, 1 \mathrm{H}), 7.08 \sim$ $7.01(\mathrm{~m}, 2 \mathrm{H}), 4.23 \sim 4.16(\mathrm{~m}, 1 \mathrm{H}), 4.10 \sim 3.99(\mathrm{~m}, 2 \mathrm{H})$, $3.98 \sim 3.87(\mathrm{~m}, 2 \mathrm{H}), 3.83(\mathrm{~s}, 3 \mathrm{H}), 3.46 \sim 3.39(\mathrm{~m}, 1 \mathrm{H})$, $(3.35 \sim 3.28,3.27 \sim 3.21,3.16 \sim 3.08)(\mathrm{m}, 2 \mathrm{H}),(1.39,1.29)$ $(\mathrm{d}, J=7.0 \mathrm{~Hz}, 3 \mathrm{H}),(1.24,0.96)(\mathrm{t}, J=7.0 \mathrm{~Hz}, 3 \mathrm{H}) ;{ }^{13} \mathrm{C}$ NMR (150 MHz, $\left.\mathrm{CDCl}_{3}\right) \delta$ : (171.6, 171.3), (157.6, 157.5), (156.6, 156.5), (136.0, 135.7), (132.8, 132.5), (128.2, 128.1), (127.8, 127.7), (126.5, 125.8), (125.6, 125.5), (125.0, 124.7), (118.0, 117.9), (104.6, 104.5), (67.4, 66.8), $(60.5,60.4),(60.2,60.0),(54.3,54.2),(46.3,46.2),(40.7$, 40.0), (18.2, 15.1), (13.2, 12.9); HRMS (ESI) calcd for $\mathrm{C}_{20} \mathrm{H}_{24} \mathrm{~N}_{2} \mathrm{NaO}_{5}[\mathrm{M}+\mathrm{Na}]^{+}$395.1577, found 395.1578.

3-(1-(4-氯苯甲酰基)-5-甲氧基-2-甲基- $1 H$-吲哚-3基)-2-((2-氧代噁唑啉-3-基)氨基)丙酸乙酯(3ay): 采用通 法 A, 柱层析洗脱剂 PE/EA $(V: V=3: 1)$; 黄色油状物 $73 \mathrm{mg}$, 产率 91\%. ${ }^{1} \mathrm{H} \mathrm{NMR}\left(600 \mathrm{MHz}, \mathrm{CDCl}_{3}\right) \delta: 7.59$ (d, $J=8.5 \mathrm{~Hz}, 2 \mathrm{H}), 7.40$ (d, $J=9.0 \mathrm{~Hz}, 2 \mathrm{H}), 6.91$ (d, $J=2.5$ $\mathrm{Hz}, 1 \mathrm{H}), 6.9$ (d, $J=9.0 \mathrm{~Hz}, 1 \mathrm{H}), 6.62(\mathrm{dd}, J=9.0,3.0 \mathrm{~Hz}$, $1 \mathrm{H}), 4.46$ (bs, 1H), $4.18 \sim 4.12(\mathrm{~m}, 2 \mathrm{H}), 4.09$ (q, $J=7.0$ $\mathrm{Hz}, 2 \mathrm{H}), 4.01(\mathrm{t}, J=7.0 \mathrm{~Hz}, 1 \mathrm{H}), 3.78(\mathrm{~s}, 3 \mathrm{H}), 3.68 \sim 3.63$ (m, 1H), 3.47 (q, $J=8.5 \mathrm{~Hz}, 1 \mathrm{H}), 3.07$ (dd, $J=14.5,6.5$ $\mathrm{Hz}, 1 \mathrm{H}), 2.93$ (dd, $J=15.0,8.0 \mathrm{~Hz}, 1 \mathrm{H}), 2.27$ (s, 3H), 1.13 $(\mathrm{t}, J=7.0 \mathrm{~Hz}, 3 \mathrm{H}) ;{ }^{13} \mathrm{C} \mathrm{NMR}\left(150 \mathrm{MHz}, \mathrm{CDCl}_{3}\right) \delta: 171.5$, $167.3,157.8,155.0,138.3,134.6,132.8,130.2,129.9$, $129.6,128.1,114.0,113.0,110.7,100.0,61.5,60.6,60.4$, 54.7, 46.7, 25.3, 13.0, 12.6; HRMS (ESI) calcd for $\mathrm{C}_{25} \mathrm{H}_{26} \mathrm{~N}_{3} \mathrm{NaO}_{6} \mathrm{Cl}[\mathrm{M}+\mathrm{Na}]^{+}$522.1402, found 522.1404.

((叔丁基二甲基硅基)氧基)缬氨酸乙酯(3da): 采用 通法 $\mathrm{B}$, 柱层析洗脱剂 $\mathrm{PE} / \mathrm{EA}(V: V=50: 1)$; 无色油状 物 $18 \mathrm{mg}$, 产率 $31 \%$. ${ }^{1} \mathrm{H} \mathrm{NMR}\left(600 \mathrm{MHz}, \mathrm{CDCl}_{3}\right) \delta$ : $4.20 \sim 4.13(\mathrm{~m}, 2 \mathrm{H}), 3.11(\mathrm{~d}, J=8.0 \mathrm{~Hz}, 1 \mathrm{H}), 1.73 \sim 1.64$ $(\mathrm{m}, 1 \mathrm{H}), 1.22$ (q, $J=7.0 \mathrm{~Hz}, 3 \mathrm{H}), 0.93(\mathrm{~d}, J=6.5 \mathrm{~Hz}, 3 \mathrm{H})$, $0.86(\mathrm{~d}, J=6.5 \mathrm{~Hz}, 3 \mathrm{H}), 0.81(\mathrm{~s}, 9 \mathrm{H}), 0.02$ (s, 3H), 0.00 (s, $3 \mathrm{H}) ;{ }^{13} \mathrm{C} \mathrm{NMR}\left(150 \mathrm{MHz}, \mathrm{CDCl}_{3}\right) \delta: 174.8,72.0,60.5$, 29.3, 26.2, 19.7, 19.5, 18.0, 14.4, -5.4, - 5.5; HRMS (ESI) calcd for $\mathrm{C}_{13} \mathrm{H}_{29} \mathrm{NNaO}_{3} \mathrm{Si}[\mathrm{M}+\mathrm{Na}]^{+}$298.1809, found 298.1808 .

化合物 5c: 采用通法 $\mathrm{C}$, 柱层析洗脱剂 PE/EA $(V$ : $V=3: 1)$; 白色固体 $17 \mathrm{mg}$, 产率 35\%. m.p. 135 $137{ }^{\circ} \mathrm{C} ;{ }^{1} \mathrm{H}$ NMR $\left(600 \mathrm{MHz}, \mathrm{CDCl}_{3}\right) \delta: 5.19$ (t, $J=3.0 \mathrm{~Hz}$, 1H), 5.03 (ddd, $J=10.5,10.5,4.0 \mathrm{~Hz}, 1 \mathrm{H}), 4.69$ (d, $J=$ 
$10.5 \mathrm{~Hz}, 1 \mathrm{H}), 4.25 \sim 4.09(\mathrm{~m}, 4 \mathrm{H}), 3.56 \sim 3.45(\mathrm{~m}, 2 \mathrm{H})$, 3.44 (s, $1 \mathrm{H}), 2.70$ (dd, $J=14.0,4.0 \mathrm{~Hz}, 1 \mathrm{H}), 2.02 \sim 1.99$ (m, 2H), 2.00 (s, 3H), 1.95 (dd, $J=12.0,4.0 \mathrm{~Hz}, 1 \mathrm{H}), 1.92$ (s, 3H), $1.89 \sim 1.78(\mathrm{~m}, 2 \mathrm{H}), 1.67(\mathrm{t}, J=13.5 \mathrm{~Hz}, 1 \mathrm{H})$, $1.63 \sim 1.56(\mathrm{~m}, 2 \mathrm{H}), 1.54 \sim 1.44(\mathrm{~m}, 2 \mathrm{H}), 1.42 \sim 1.29(\mathrm{~m}$, $2 \mathrm{H}), 1.27 \sim 1.17(\mathrm{~m}, 1 \mathrm{H}), 1.22(\mathrm{t}, J=13.0 \mathrm{~Hz}, 3 \mathrm{H}), 1.11(\mathrm{~s}$, $3 \mathrm{H}), 1.11 \sim 1.08(\mathrm{~m}, 1 \mathrm{H}), 1.07 \sim 1.01(\mathrm{~m}, 3 \mathrm{H}), 1.01(\mathrm{~s}$, $3 \mathrm{H}), 0.98(\mathrm{~d}, J=12.5 \mathrm{~Hz}, 1 \mathrm{H}), 0.94 \sim 0.91(\mathrm{~m}, 1 \mathrm{H}), 0.92$ $(\mathrm{s}, 3 \mathrm{H}), 0.90 \sim 0.87(\mathrm{~m}, 1 \mathrm{H}), 0.86(\mathrm{~s}, 3 \mathrm{H}), 0.85(\mathrm{~s}, 3 \mathrm{H})$, $0.83(\mathrm{~s}, 3 \mathrm{H}), 0.81$ (s, 3H); ${ }^{13} \mathrm{C}$ NMR $\left(150 \mathrm{MHz}, \mathrm{CDCl}_{3}\right) \delta$ : $171.9,169.9,169.6,157.4,143.4,121.6,79.5,69.0,65.5$, $60.4,59.8,53.7,46.4,46.3,45.3,42.9,40.3,40.2,38.8$, $38.3,38.2,36.9,32.9,32.0,30.9,29.8,27.4,25.4,25.2$, 24.1, 22.7, 22.6, 22.3, 20.2, 19.9, 17.1, 16.6, 15.6, 15.5, 13.2; HRMS (ESI) calcd for $\mathrm{C}_{40} \mathrm{H}_{62} \mathrm{~N}_{2} \mathrm{NaO}_{8}[\mathrm{M}+\mathrm{Na}]^{+}$ 721.4398 , found 721.4394 .

\section{辅助材料(Supporting Information) 新化合物 3af、} 3ap、3aq、3ar、3as、3at、3au、3av、3da、5c 的 ${ }^{1} \mathrm{H} N \mathrm{NR}$ 和 ${ }^{13} \mathrm{C}$ NMR 谱图. 这些材料可以免费从本刊网站(http:// sioc-journal.cn/)上下载.

\section{References}

[1] Liu, Q.; Wu, L. Z. Nat. Sci. Rev. 2017, 4, 359.

[2] (a) Marzo, L.; Pagire, S. K.; Reiser, O.; König, B. Angew. Chem., Int. Ed. 2018, 57, 10034.

(b) Shaw, M. H.; Twilton, J.; MacMillan, D. W. C. J. Org. Chem. 2016, 81, 6898 .

[3] (a) Song, H.; Liu, X. Y.; Qin, Y. Acta Chim. Sinica 2017, 75, 1137 (in Chinese).

(宋影, 刘小宇, 秦勇, 化学学报, 2017, 75, 1137.)

(b) Song, C. H.; Shen, X.; Yu, F.; He, Y. P.; Yu, S. Y. Chin. J. Org. Chem. 2020, 40, 3748 (in Chinese).

(宋常华, 沈许, 于芳, 何宇鹏, 俞寿云, 有机化学, 2020, 40, 3748.)

(c) Xiao, L.; Li, J. H.; Wang, T. Acta Chim. Sinica 2019, 77, 841 (in Chinese).

(肖丽, 李嘉恒, 王挺, 化学学报, 2019, 77, 841.)

(d) Kong, Y. L.; Xu, W. X.; Ye, F. X.; Weng, J. Q. Chin. J. Org. Chem. 2019, 39, 3065 (in Chinese).

(孔瑶蕾, 徐雯秀, 叶飞霞, 翁建全, 有机化学, 2019, 39, 3065.)

[4] Yan, M.; Lo, J. C.; Edwards, J. T.; Baran, P. S. J. Am. Chem. Soc. 2016, 138, 12692.

[5] (a) Romero, N. A.; Nicewicz, D. A. Chem. Rev. 2016, 116, 10075. (b) Bogdos, M. K.; Pinard, E.; Murphy, J. A. Beilstein J. Org. Chem. 2018, 14, 2035.

[6] Twilton, J.; Le, C.; Zhang, P.; Shaw, M. H.; Evans R. W.; MacMillan, D. W. C. Nat. Rev. Chem. 2017, 1, 0052.

[7] For recent reviews, see:

(a) Crisenza, G. E. M.; Mazzarella, D.; Melchiorre, P. J. Am. Chem. Soc. 2020, 142, 5461 .

(b) Lima, C. G. S.; Lima, T. d. M.; Duarte, M.; Jurberg, I. D.; Paixão, M. W. ACS Catal. 2016, 6, 1389.

(c) Yuan, Y.-Q.; Majumder, S.; Yang, M.-H.; Guo, S.-R. Tetrahe- dron Lett. 2020, 61, 151506

[8] For recent examples, see:

(a) Pitre, S. P.; Allred, T. K.; Overman, L. E. Org. Lett. 2021, 23, 1103.

(b) Xia, Q.; Li, Y. F.; Cheng, L.; Liang, X.; Cao, C. L.; Dai, P.; Deng, H. P.; Zhang, W. H.; Wang, Q. M. Org. Lett. 2020, 22, 9638. (c) Xie, S. S.; Li, D. F.; Huang, H. C.; Zhang, F. Y.; Chen, Y. Y. J. Am. Chem. Soc. 2019, 141, 16237.

(d) Liang, K. J.; Li, N.; Zhang, Y.; Li, T.; Xia, C. F. Chem. Sci. 2019, 10, 3049.

(e) Zhang, H. H.; Yu, S. Y. Org. Lett. 2019, 21, 3711.

(f) Cao, Z. Y.; Ghosh, T.; Melchiorre, P. Nat. Commun. 2018, 9, 3274.

(g) Guo, Q. P.; Wang, M. R.; Liu, H.; Wang, R.; Xu, Z. Q. Angew. Chem., Int. Ed. 2018, 57, 4747 .

(h) Fawcett, A.; Pradeilles, J.; Wang, Y.; Mutsuga, T.; Myers, E. L.; Aggarwal, V. K. Science 2017, 357, 283.

[9] Fu, M. C.; Shang, R.; Zhao, B.; Wang, B.; Fu, Y. Science 2019, 363, 1429.

[10] Jiao, M. J.; Liu, D.; Hu, X. Q.; Xu, P. F. Org. Chem. Front. 2019, 6, 3834.

[11] Wadekar, K.; Aswale, S.; Yatham, V. R. RSC Adv. 2020, 10, 16510.

[12] Wang, Y. T.; Fu, M. C.; Zhao, B.; Shang, R.; Fu, Y. Chem. Commun. 2020, 56, 2495.

[13] Wang, H. Y.; Zhong, L. J.; Lü, G. F.; Li, Y.; Li, J. H. Org. Biomol. Chem. 2020, 18, 5589.

[14] Liu, H. Y.; Lu, Y.; Li, Y.; Li, J. H. Org. Lett. 2020, 22, 8819.

[15] (a) Sheng, H.; Liu, Q.; Su, X. D.; Lu, Y.; Wang, Z. X.; Chen, X. Y. Org. Lett. 2020, 22, 7187.

(b) Zhang, C. S.; Bao, L.; Chen, K. Q.; Wang, Z. X.; Chen, X. Y. Org. Lett. 2021, 23, 1577.

[16] For recent reviews, see:

(a) Friestad, G. K. Top. Curr. Chem. 2014, 343, 1.

(b) Friestad, G. K. Top. Curr. Chem. 2012, 320, 61.

[17] Garrido-Castro, A. F.; Maestro, M. C.; Alemán, J. Catalysts 2020, 10,562 .

[18] For recent examples:

(a) Jia, J. Q.; Lefebvre, Q.; Rueping, M. Org. Chem. Front. 2020, 7 , 602.

(b) Pantaine, L. R. E.; Milligan, J. A.; Matsui, J. K.; Kelly, C. B.; Molander, G. A. Org. Lett. 2019, 21, 7, 2317.

(c) Cullen, S. T. J.; Friestad, G. K. Org. Lett. 2019, 21, 8290

(d) Ji, P.; Zhang, Y. T.; Wei, Y. Y.; Huang, H.; Hu, W. B.; Mariano, P. A.; Wang, W. Org. Lett. 2019, 21, 3086.

(e) Garrido-Castro, A. F.; Choubane, H.; Daaou, M.; Maestro, M. C.; Alemán, J. Chem. Commun. 2017, 53, 7764.

[19] For recent examples:

(a) Weigel, W. K., III.; Dang, H. T.; Yang, H. B.; Martin, D. B. C. Chem. Commun. 2020, 56, 9699.

(b) Jia, J. Q.; Kancherla, R.; Rueping, M.; Huang, L. Chem. Sci. 2020, 11, 4954.

(c) Yang, S.; Zhu, S. Y.; Lu, D. F.; Gong, Y. F. Org. Lett. 2019, 21, 8464.

[20] For recent examples:

(a) Leitch, J. A.; Rogova, T.; Duarte, F.; Dixon, D. J. Angew. Chem., Int. Ed. 2020, 59, 4121.

(b) Rong, J. W.; Seeberger, P. H.; Gilmore, K. Org. Lett. 2018, 20 , 4081.

(c) Chen, M.; Zhao, X. X.; Yang, C.; Xia, W. J. Org. Lett. 2017, 19, 3807.

(d) Qi, L.; Chen, Y. Y. Angew. Chem., Int. Ed. 2016, 55, 13312.

(e) Uraguchi, D.; Kinoshita, N.; Kizu, T.; Ooi, T. J. Am. Chem. Soc. 
2015, 137,13768

(f) Jeffrey, J. L.; Petronijević, F. R.; MacMillan, D. W. C. J. Am. Chem. Soc. 2015, 137, 8404.

[21] Tang, R.; Shao, Z. Y.; Wang, J. C.; Liu, Z. X.; Li, Y. M.; Shen, Y. H. J. Org. Chem. 2019, 84, 8177.

[22] Wang, J. C.; Shao, Z. Y.; Tan, K.; Tang, R.; Zhou, Q. L.; Xu, M.; Li, Y. M.; Shen, Y. H. J. Org. Chem. 2020, 85, 9944.

[23] (a) Jung, J.; Kim, J.; Park, G.; You, Y.; Cho, E. J. Adv. Synth. Catal.
2016, 358, 74 .

(b) Chen, W. X.; Tao, H. C.; Huang, W. H.; Wang, G. Q.; Li, S. H.; Cheng, X.; Li, G. G. Chem.-Eur. J. 2016, 22, 9546.

(c) Zhang, J.; Li, Y.; Xu, R. Y.; Chen, Y. Y. Angew. Chem., Int. Ed. 2017, 56, 12619.

(d) Yu, J.; Lin, J. H.; Cao, Y. C.; Xiao, J. C. Org. Chem. Front. 2019, 6, 3580 . 